\title{
Low Reynolds Number Aerodynamics of Low-Aspect-Ratio, Thin/Flat/Cambered-Plate Wings
}

\author{
Alain Pelletier* and Thomas J. Mueller ${ }^{\dagger}$ \\ University of Notre Dame, Notre Dame, Indiana 46556
}

\begin{abstract}
The design of micro aerial vehicles requires a better understanding of the aerodynamics of small low-aspectratio wings. An experimental investigation has focused on measuring the lift, drag, and pitching moment about the quarter chord on a series of thin flat plates and cambered plates at chord Reynolds numbers varying between 60,000 and 200,000. Results show that the cambered plates offer better aerodynamic characteristics and performance. It also appears that the trailing-edge geometry of the wings and the turbulence intensity in the wind tunnel do not have a strong effect on the lift and drag for thin wings at low Reynolds numbers. Moreover, the results did not show the presence of any hysteresis, which is usually observed with thick airfoils/wings.
\end{abstract}

$\mathrm{AR}$
$a$
$C_{D}$
$C_{d}$
$C_{L}$
$C_{l}$
$C_{L \alpha}$
$C_{L}^{3 / 2} / C_{D}$
$C_{m / 4}$
$C_{m_{\alpha}}$
$c$
$e_{Q}$
$L / D$
$M$
$R e_{c}$
$\mathrm{sAR}$
$t$
$\alpha$
$\alpha C_{L}=0$
$\alpha_{\text {stall }}$
$\tau$

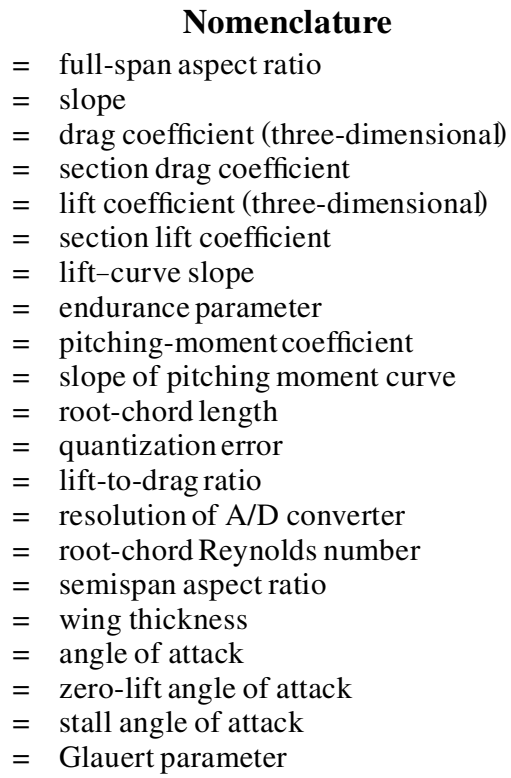

\section{Subscripts}

$\max \quad=$ maximum

$\min =$ minimum

$0=$ two-dimensional

\section{Introduction}

I $\mathrm{N}$ this paper we present some of the results of an experimental investigation on low Reynolds number aerodynamics of small low-aspect-ratio wings. Recently, the need for small micro aerial vehicles (MAVs) has surfaced. These MAVs would have a wing span of no more than 6 in. $(15.2 \mathrm{~cm})$ and weigh only a few ounces $(\approx 100-200$ g) (Ref. 1). They could be used as reconnaissance vehicles and carry visual, acoustic, chemical, or biologicalsensors. They should be able to fly for $20 \mathrm{~min}$ to $2 \mathrm{~h}$ at a maximum speed of up to $30 \mathrm{mph}(48 \mathrm{~km} / \mathrm{h})$. For these vehicles, root-chord Reynolds numbers ranging from $\sim 2 \times 10^{4}$ to $\sim 2 \times 10^{5}$ are of interest. Very little data

Received 26 October 1999; revision received 21 March 2000; accepted for publication 26 March 2000. Copyright (C) 2000 by Alain Pelletier and Thomas J. Mueller. Published by the American Institute of Aeronautics and Astronautics, Inc., with permission.

${ }^{*}$ Postdoctoral Research Assistant, Department of Aerospace and Mechanical Engineering. Member AIAA.

${ }^{\dagger}$ Roth-Gibson Professor, Department of Aerospace and Mechanical Engineering. Associate Fellow AIAA. exist for fixed low-aspect-ratiowings in this range. Selig et al. ${ }^{2}$ have looked at the aerodynamic characteristics of a series of airfoils at Reynolds numbers from approximately $6 \times 10^{4}$ to $3 \times 10^{5}$, including a $2 \%$ thick flat-plate airfoil. The results were very promising; unfortunately, no data were presented for small-aspect-ratio wings. This led to force balance tests being conducted at the Hessert Center for Aerospace Research at the University of Notre Dame on a series of small flat and cambered plates of low aspect ratio at different Reynolds numbers. For simplicity, the aspect ratio mentioned in this paper is the semispan aspect ratio, unless indicated otherwise.

For all the wings tested, the behavior of $C_{L}, C_{D}, C_{m / 4}, L / D$, and $C_{L}^{3 / 2} / C_{D}$ was sought. For convenience in the text, $C_{L}$ and $C_{D}$ will refer to both two-dimensionalairfoildata and three-dimensional wing data. By conducting tests at different Reynolds numbers, it was possible to study the influence of Reynolds number and aspect ratio on the aerodynamic characteristics of small low-aspect-ratio wings.

All results presented in this paper have been corrected for solid blockage, wake blockage, and streamlined curvature by use of techniques presented by Pankhurst and Holder ${ }^{3}$ and Rae and Pope. ${ }^{4}$ Because of the small thickness and the volume of the wings tested, the effect of blockage was very limited.

\section{Apparatus}

\section{Wind Tunnel}

Tests presented in this paper were conducted in a low-speed, lowturbulence wind tunnel. The tunnel had a test section with a $2 \times 2 \mathrm{ft}$ $(61 \times 61 \mathrm{~cm})$ cross section. The range of velocities required for tests between $R e_{c}=6 \times 10^{4}$ and $2 \times 10^{5}$ could easily be obtained in the wind tunnel. The freestreamturbulenceintensity was approximately $0.05 \%$ over the range of interest.

Aluminum end plates were mounted in the test section. The bottom plate could be removed to simulate a semi-infinite model. All wings tested were held at the quarter-chord point, and the sting was covered by a streamlined sting covering. The gaps between the wing and the end plates were adjusted to approximately $0.03 \mathrm{in} .(0.8 \mathrm{~mm})$. Mueller and Burns ${ }^{5}$ showed that gap sizes varying between 0.1 and $1.4 \mathrm{~mm}$ are usually acceptable and do not affect the results. Moreover, Rae and Pope ${ }^{4}$ suggest that the gap be less than $0.005 \times$ span. For a 12-in.-(30.5-cm-) span model, this correspondsto a maximum gap size of 0.06 in. $(1.5 \mathrm{~mm})$, which is larger than the gaps used in the current investigation.

\section{Water Tunnel}

A free-surface water tunnel with a test-section cross section of $15 \times 18$ in. $(38.1 \times 45.7 \mathrm{~cm})$ was used for flow visualization. Water velocities up to $1.28 \mathrm{ft} / \mathrm{s}(39 \mathrm{~cm} / \mathrm{s})$ can be obtained in the test section. A freestream turbulence intensity of less than $1 \%$ has been reported by the manufacturer of the water tunnel. This facility is excellent 
Table 1 Wing dimensions

\begin{tabular}{lccccc}
\hline \hline Designation $^{\mathrm{a}}$ & Chord,in. & Span, in. & sAR & Thickness, in. & Camber, \% \\
\hline C8S4 & 7.973 & 3.998 & 0.5 & 0.155 & 0 \\
C8S8 & 7.973 & 8.003 & 1.0 & 0.154 & 0 \\
C8S12 & 7.985 & 12.01 & 1.5 & 0.157 & 0 \\
C4S8 & 3.999 & 8.019 & 2.0 & 0.077 & 0 \\
C4S12 & 4 & 12.014 & 3.0 & 0.077 & 0 \\
C8S4C & 7.975 & 3.995 & 0.5 & 0.156 & 4 \\
C8S8C & 7.983 & 8 & 1.0 & 0.156 & 4 \\
C8S12C & 7.908 & 12.013 & 1.5 & 0.156 & 4 \\
C4S8C & 3.995 & 8 & 2.0 & 0.078 & 4 \\
C4S12C & 3.936 & 11.998 & 3.0 & 0.079 & 4 \\
C8S12E & 7.969 & 12.011 & 1.5 & 0.156 & 0 \\
C8S12CE & 7.931 & 12.011 & 1.5 & 0.157 & 4 \\
\hline \hline
\end{tabular}

${ }^{\mathrm{a}} \mathrm{C}$, cambered; E, elliptical trailing edge.

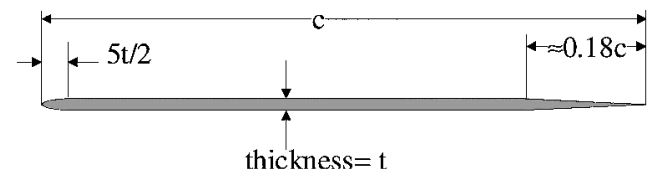

a) Flat plate

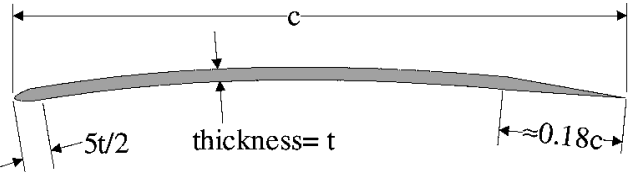

b) Cambered plate

Fig. 1 Airfoil geometry for models with tapered trailing edge.

for flow visualizationby either the hydrogen-bubbleor dye-injection technique.In this investigation, hydrogen-bubbleflow visualization was conducted in order to study flow separation.

\section{Models}

For this investigation, several thin, flat, and cambered rectangular aluminum models with a thickness-to-chord ratio of $1.93 \%$ were built. Thin models were selected because birds, who glide at low Reynolds numbers, have very thin wings. The models had either a 5-to-1 elliptical leading edge and a 3-deg tapered trailing edge or a 5-to-1 elliptical leading edge and trailing edge. The cambered models had a circular arc shape with $4 \%$ camber. The semispan aspect ratios tested varied between 0.50 and 3.00. The dimensions of the models built for this study are presented in Table 1. Figure 1 shows schematics of the airfoil geometries for the wings with a tapered trailing edge.

\section{Force Balance}

Description

All results presented in this paper were obtained with a threecomponent platform force balance. This balance was used to measure lift, drag, and pitching moment about the vertical axis. The balance is an external balance placed on top of the test section. With this balance, lift and drag forces are transmitted through the sting that is mounted directly to the moment sensor. The moment sensor is rigidly mounted to an adjustable-angle-ofattack mechanism on the top platform. The lift platform is supported from a platform, called the drag platform, by two vertical plates that flex in only the lift direction. The lift and drag platforms are also connected with a flexure with bonded-foil strain gauges mounted on it. The drag platform is supported by two vertical plates that flex in only the drag direction and hang from two more vertical flexible plates attached to the base platform of the balance. The base and drag platforms are also connected by a flexure with strain gauges mounted on it. Both flexures act like cantilever beams when loads are applied to the balance. A second set of flexures, for both lift and drag, can be engaged when the loads are large. For the range of forces mea-

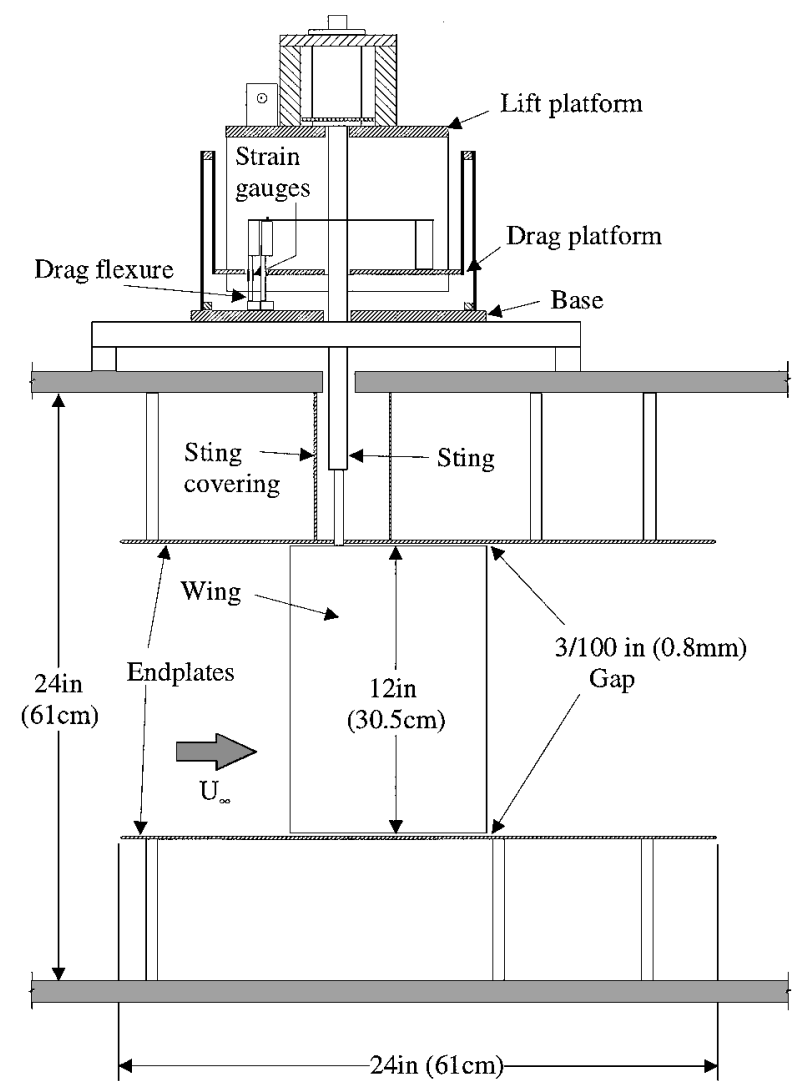

Fig. 2 Balance arrangement in the test section.

sured in this investigation (up to $\sim 11 \mathrm{~N}$ in lift and $5 \mathrm{~N}$ in drag), the second set of flexures was never engaged. Figure 2 shows a schematic of the balance setup in the test section. The maximum forces that can be measured with the balance without engaging the second set of flexures are $14.7 \mathrm{~N}$ for lift and drag and $226 \mathrm{~N} \cdot \mathrm{cm}$ for the pitching moment, ${ }^{6}$ whereas the minimum measurable loads are approximately $0.01 \mathrm{~N}$ for lift and drag and $0.05 \mathrm{~N} \cdot \mathrm{cm}$ for the moment. A complete description of the balance was presented by Mueller et al. ${ }^{7}$ A calibration of the balance was performed before each series of tests, and the results showed a linear behavior for all three axes: lift, drag, and moment. The calibrations were repeatable from one day to another. Finally, the uncertainty in the aerodynamic coefficients obtained from the force balance will be discussed below.

\section{Data-Acquisition System}

The strain gauges from the force balance were configured in full Wheatstonebridges. An excitation voltage of $5 \mathrm{~V}$ was used for all the strain-gauge bridges. The bridge signals were read with an instrumentation amplifier circuit. The amplified analog signals were sent to the computer where they were then converted by a four-channel, 12-bit A/D converter. Four data channels (lift, drag, moment, and dynamic pressure) could be measured quasi-simultaneously. All the data were acquired with a PC-based data-acquisitionsystem running the LABVIEW ${ }^{\circledR} 5$ graphical programming language. Throughout the tests, the aerodynamic coefficients were obtained by the averaging of 4000 samples acquired at a frequency of $500 \mathrm{~Hz}$.

\section{Uncertainty}

The uncertainties in the results presented next were determined with the Kline-McClintock technique ${ }^{8}$ for error propagation. The two main sources of uncertainty were the quantization error and the uncertainty arising from the standard deviation of a given mean output voltage from the strain gauges of the force balance. The quantization error is $e_{Q}=\frac{1}{2}$ [Range in volts $/ 2^{M}$ ]. Optimizing the range of the output voltages can help to reduce the uncertainties. 


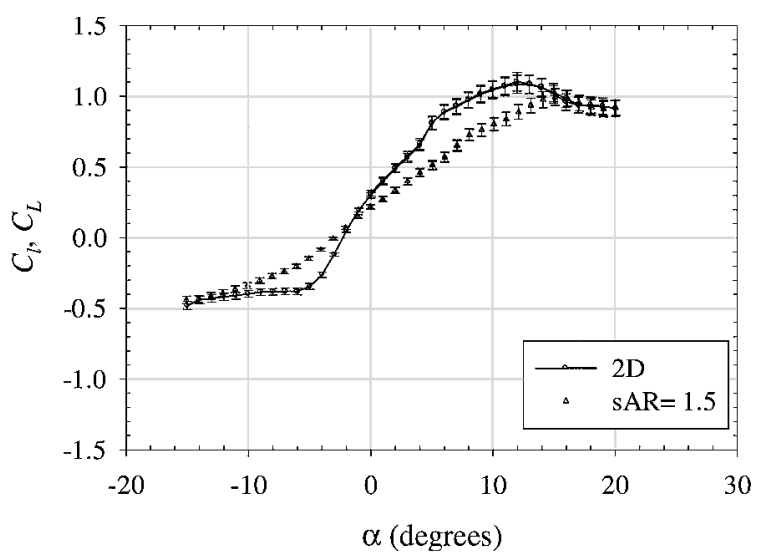

a) Lift coefficient

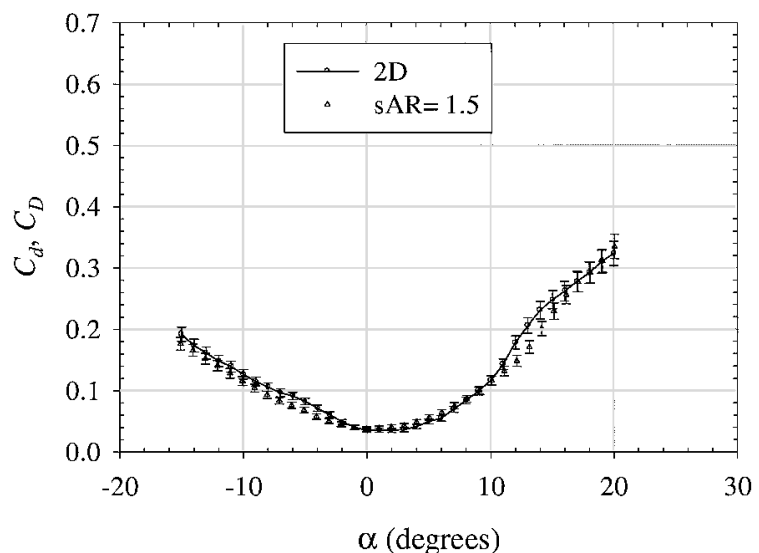

b) Drag coefficient

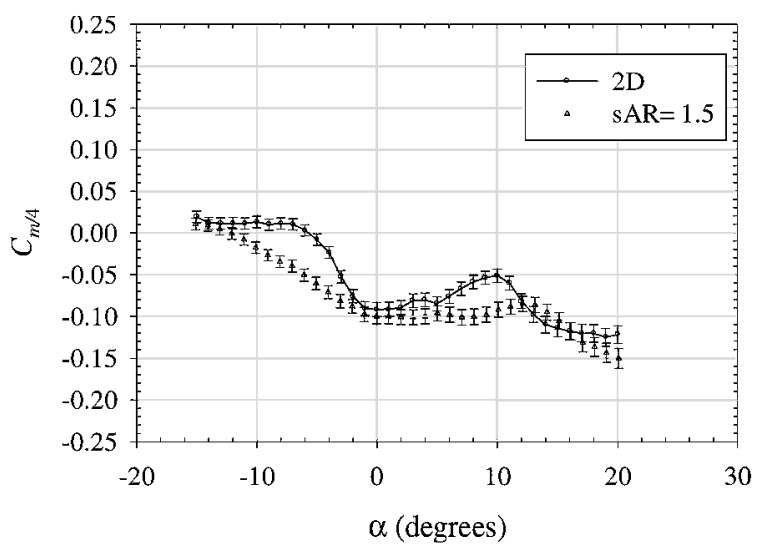

c) Pitching-moment coefficient

Fig. 3 Uncertainties in aerodynamic coefficients for cambered plates at $R e_{c}=6 \times 10^{4}$.

If the gain is increased, the standard deviation of the mean will also be increased, but the ratio of the standard deviation to the mean will basically remain the same. However, the uncertainty from the quantization error will be reduced because the quantization error is a fixed value (a function of the range and the resolution of the A/D converter). The ratio of the quantization error to the mean voltage will then be smaller if a larger gain is used and a larger balance output mean voltage is obtained.

The uncertainty in the angle of attack was determined to be of the order of 0.2-0.3 deg. Figure 3 shows an example of uncertainties obtained at $R e_{c}=6 \times 10^{4}$ with the cambered plates. Error bars indicate the uncertainty in $C_{L}, C_{D}$, and $C_{m / 4}$. The uncertainties are approximately $6 \%$ for $C_{L}$ and $C_{D}$ and $10 \%$ for $C_{m / 4}$. These values were typical throughout the measurements.

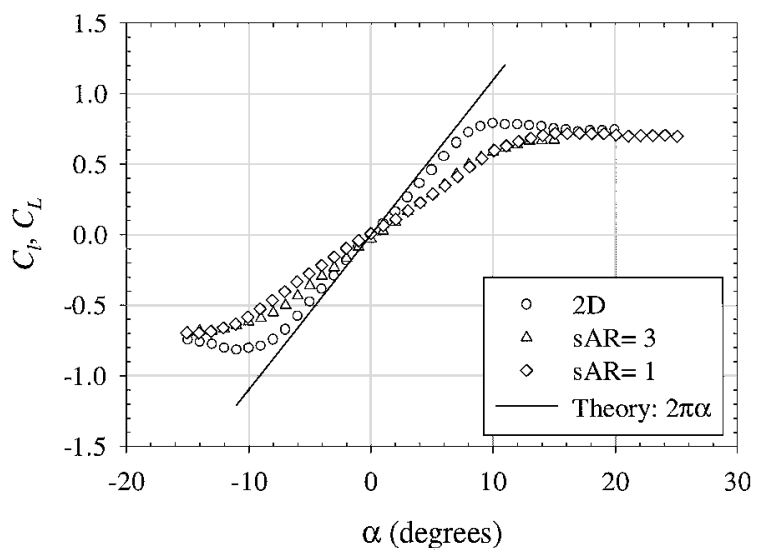

a) Lift coefficient

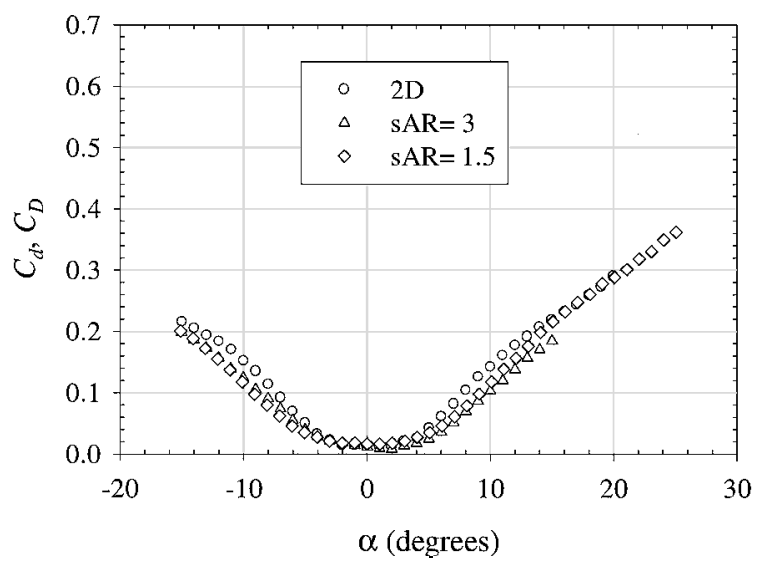

b) Drag coefficient

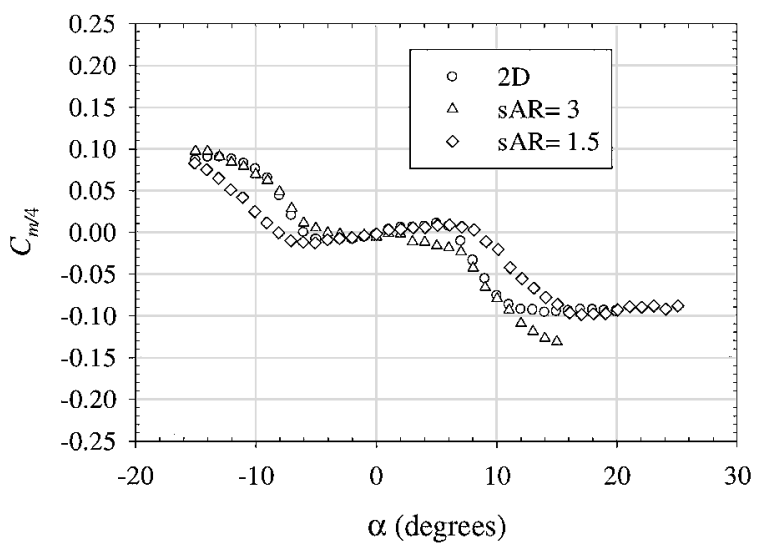

c) Pitching-moment coefficient

Fig. 4 Flat-plate characteristics at $R e_{c}=8 \times 10^{4}$.

\section{Discussion of Results}

\section{Results for Flat-Plate Wings}

Some results for the flat-plate models can be seen in Figs. 4 and 5 for $R e_{c}=8 \times 10^{4}$ and $1.4 \times 10^{5}$, respectively. It was found that no hysteresis was present in the data. Qualitative flow visualization was conducted in a water tunnel by use of hydrogen bubbles in order to observe the behavior of the boundary layer for the $\mathrm{sAR}=1$ and the $\mathrm{sAR}=0.5$ models. It was found that there was a thin region of separated flow on the suction surface near the trailing edge at low angles of attack. This region increased to more than $50 \%$ of the chord after $\alpha=8 \mathrm{deg}$. The water-tunnel flow visualizationindicated that there was never any flow reattachment after separation, which may explain the lack of hysteresis. It could also be related to the fact that none of the data indicated a sharp stall (abrupt reduction in $C_{L}$ ), which is usually seen when hysteresisoccurs. The leading-edge 


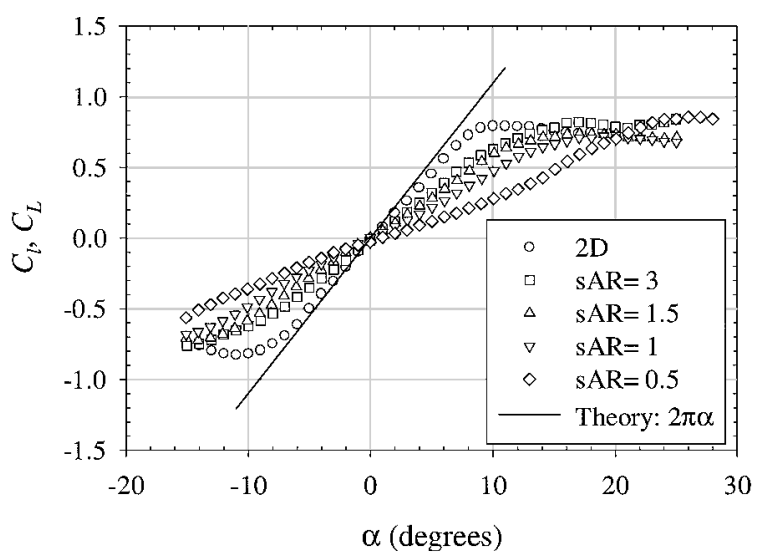

a) Lift coefficient

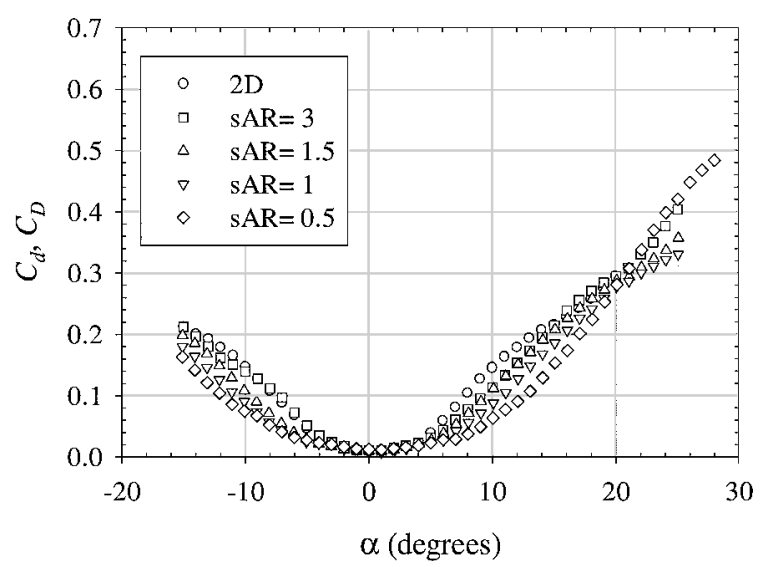

b) Drag coefficient

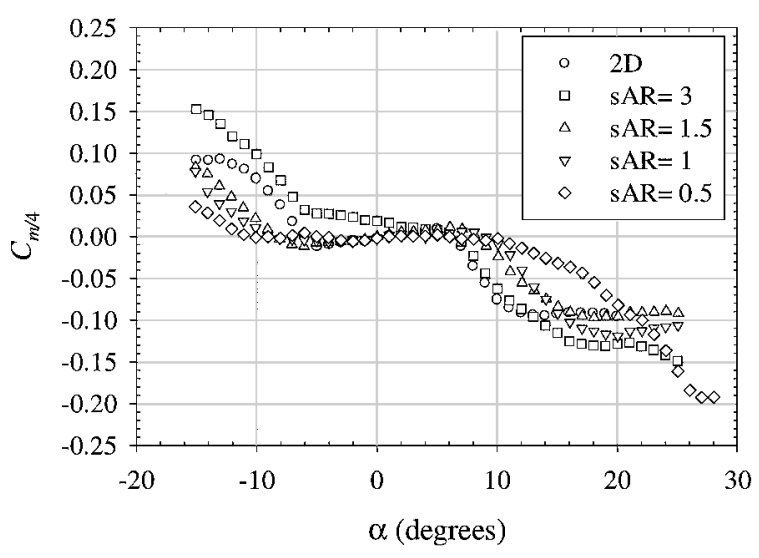

c) Pitching-moment coefficient

Fig. 5 Flat-plate characteristics at $R e_{c}=1.4 \times 10^{5}$.

geometry did not appear to be a factor in the lack of hysteresis for these thin wings because the lift and drag results ( $\mathrm{sAR}=1.5$ and two-dimensional) for an inverted model (sharp leading edge and elliptical trailing edge) were the same as those for the model with an elliptical leading edge and a tapered trailing edge. The effect of the trailing-edge geometry on hysteresis was also negligible, and is presented in the subsequent subsection on trailing-edge geometry. Unpublished data from undergraduate student work at Notre Dame for a $5.2 \%$ thick flat plate also showed the lack of hysteresis at $R e=8 \times 10^{4}$ and $1.7 \times 10^{5}$. Because no hysteresis was observed in the static results, only data for increasing angles of attack are presented. Figures $4 \mathrm{a}$ and $5 \mathrm{a}$ show a significant reduction in the lift-curve slope $C_{L_{\alpha}}$ for a finite wing. The lift-curve slope values obtained from the wind-tunnel data are compared with theoretical values for thin wings of different aspect ratios in Fig. 6.

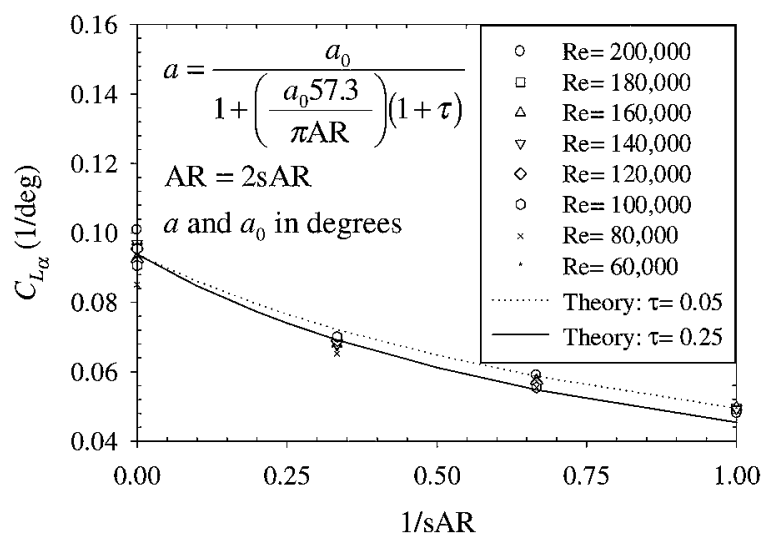

Fig. 6 Lift-curve slope for flat-plate models.

Equation 1 from Anderson ${ }^{9}$ was used to estimate the theoretical values of $C_{L_{\alpha}}$ :

$$
C_{L \alpha}=a=a_{0} /\left[1+\left(\frac{a_{0} 57.3}{\pi \mathrm{AR}}\right)(1+\tau)\right]
$$

where $\mathrm{AR}=2 \mathrm{sAR}$ and $\tau$ typically varies between 0.05 and 0.25 . The lift-curve slopes in the linear region for the two-dimensional experimental data at all Reynolds numbers tested were averaged to get an estimate of $a_{0}$. This value was determined to be $a_{0}=0.0938 / \mathrm{deg}$. This value, based on experimental results, was used instead of the conventional value of $a_{0}=2 \pi / \mathrm{rad}=0.1 / \mathrm{deg}$ given by thin-airfoil theory. Figure 6 shows very good agreement between the experimental values of $C_{L_{\alpha}}$ and the theoretical values estimated by Eq. (1).

The expected reduction in the lift-curve slope with decreasing aspect ratio (increasing 1/sAR) is obvious. Furthermore, as the aspect ratio was decreased, Figures $4 \mathrm{a}$ and $5 \mathrm{a}$ also show that the linear region of the lift coefficient vs $\alpha$ curve became longer and $\alpha_{\text {stall }}$ increased. Moreover, both figures show that there was no abrupt stall for low-aspect-ratio wings. For these low aspect ratios, the lift coefficient often reached a plateau and then remained relatively constant, or even started to increase, for increasing angles of attack. As mentioned earlier, this may be associated with the lack of hysteresis.

The lift coefficient data for the two-dimensional model agreed very well with data presented by Selig et al. ${ }^{2}$ for a $2 \%$ flat plate at $R e=1.02 \times 10^{5}$. The maximum lift coefficient was very close to that obtained in this investigation, as will be shown later when we look at performance characteristics. Moreover, the linear region for their data extended up to $8 \mathrm{deg}$ with a slope of $0.092 / \mathrm{deg}$, consistent with the results presented in this paper. Changing the aspect ratio of the models did not appear to have a strong effect on the drag coefficient at $R e_{c}=8 \times 10^{4}$, as shown in Fig. 4b. At $R e_{c}=1.4 \times 10^{5}$, increasing the aspect ratio had the unexpected effect of increasing $C_{D}$ for angles greater than $5 \mathrm{deg}$. No measurable difference was encountered in the range $-5 \mathrm{deg} \leq \alpha \leq 5 \mathrm{deg}$.

Finally, Figs. $4 \mathrm{c}$ and $5 \mathrm{c}$ show the pitching moment at the quarter chord. Both figures indicate a slightly positive slope $C_{m_{\alpha}}$ at approximately $\alpha=0 \mathrm{deg}$, even when the uncertainty is considered. This would imply that the flat-plate models were statically unstable around $\alpha=0$ deg. Increasing the Reynolds number from $8 \times 10^{4}$ to $1.4 \times 10^{5}$ reduced the slope of $C_{m / 4}$.

\section{Results for Cambered-Plate Wings}

Results were also obtained for cambered-plate models. In general, camber led to better aerodynamic characteristicsbecause of an increase in lift, even though drag also increased. Water-tunnel flow visualizationindicated that the flow on the cambered platesremained attached longer over much of the airfoil (up to $\alpha=16 \mathrm{deg}$ ) than for the flat plates. For sAR $=1, \alpha=16$ deg is close to the stall angle. Figures 7 and 8 show some results for the cambered plates, which also did not show any hysteresis, at $R e_{c}=6 \times 10^{4}$ and $1.4 \times 10^{5}$ respectively. With cambered plates, $C_{D_{\min }}$ was slightly larger than 


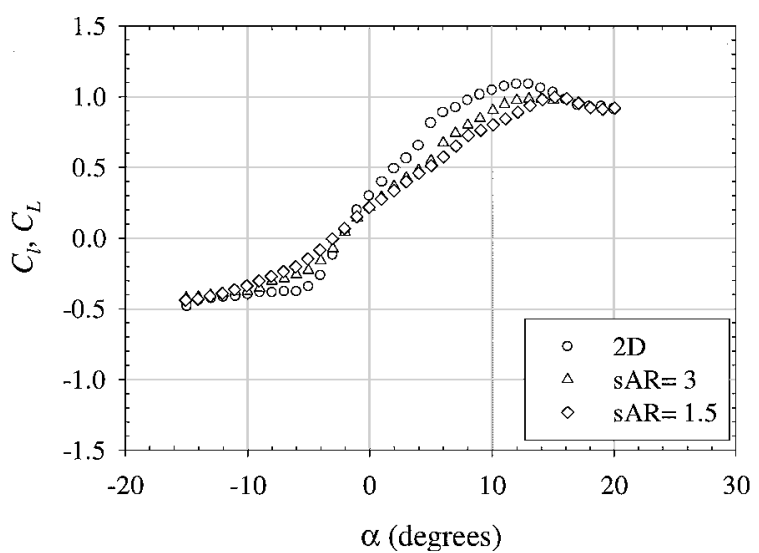

a) Lift coefficient

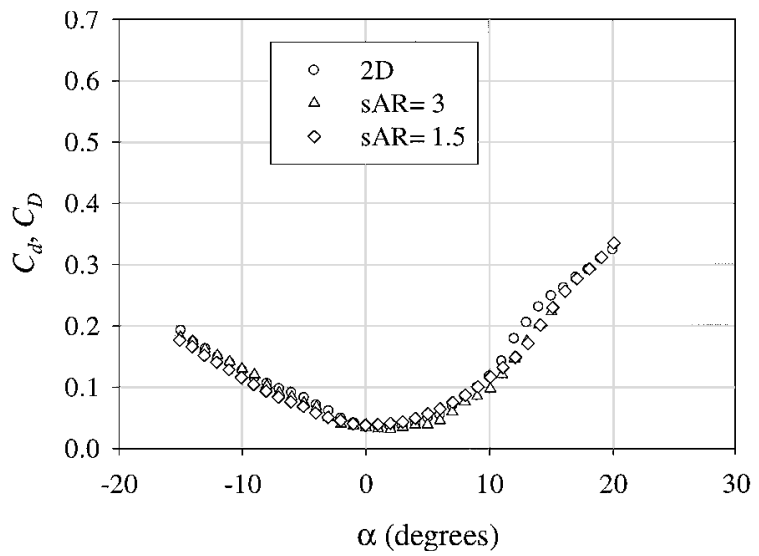

b) Drag coefficient

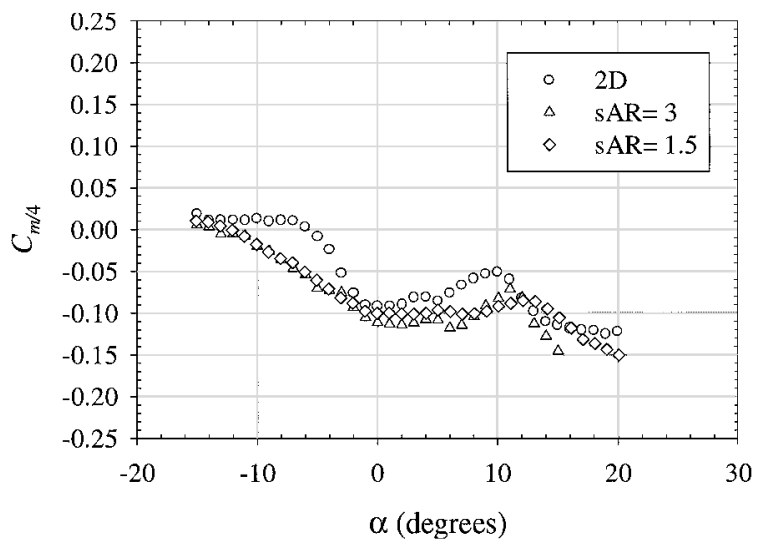

c) Pitching-moment coefficient

Fig. 7 Cambered-plate characteristics at $R e_{c}=6 \times 10^{4}$.

for flat plates. The maximum lift coefficient was also larger, as expected. Moreover, the variation in the lift coefficient with angle of attack at small angles was less linear for cambered plates than for flat plates. Finally, the behavior of the moment coefficient $C_{m / 4}$ for the cambered plates was very different than the behavior with the flat plates. A rise in $C_{m / 4}$ occurred after $\alpha \approx 5 \mathrm{deg}$, leading to a hump at approximately $\alpha \approx 10 \mathrm{deg}$. This was not observed with the flat plates.

Equation (1) was also used to compare the experimental values of $C_{L_{\alpha}}$ at $\alpha_{C_{L}}=0$ for the cambered plates to theoretical values. The two-dimensionalvalue $a_{0}$ used was $a_{0}=0.1097 / \mathrm{deg}$. Figure 9 shows a good agreement between theory and experiments.

Analysis of the results indicated that the aerodynamic characteristics of cambered plates were superior to those of flat plates, especially the maximum lift coefficient and the maximum $L / D$ ratio, as can be seen in Figs. 10-13, where two-dimensional results for flat

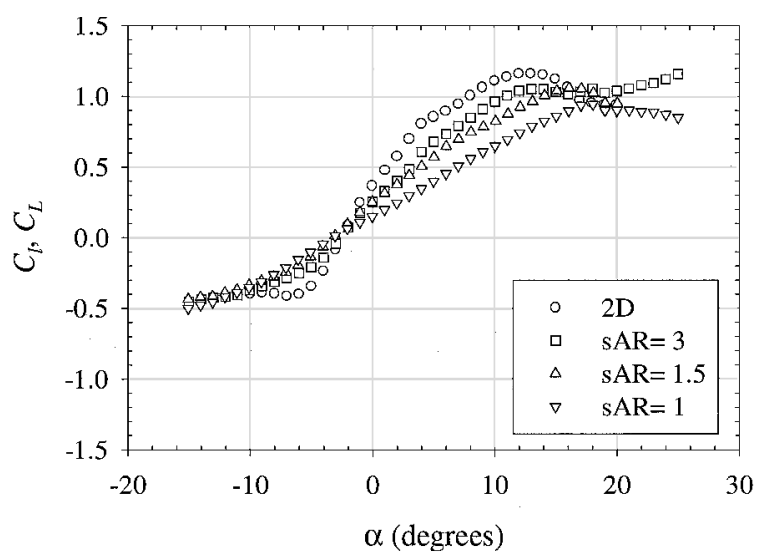

a) Lift coefficient

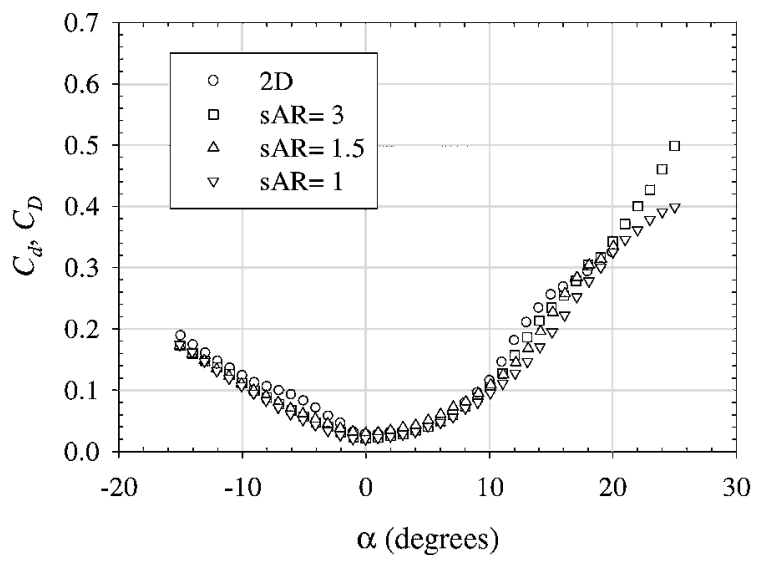

b) Drag coefficient

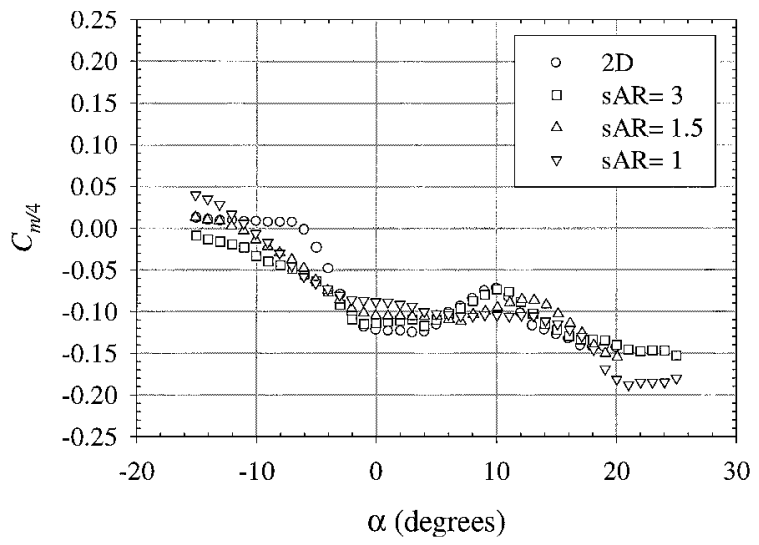

c) Pitching-moment coefficient

Fig. 8 Cambered-plate characteristics at $R e_{c}=1.4 \times 10^{5}$.

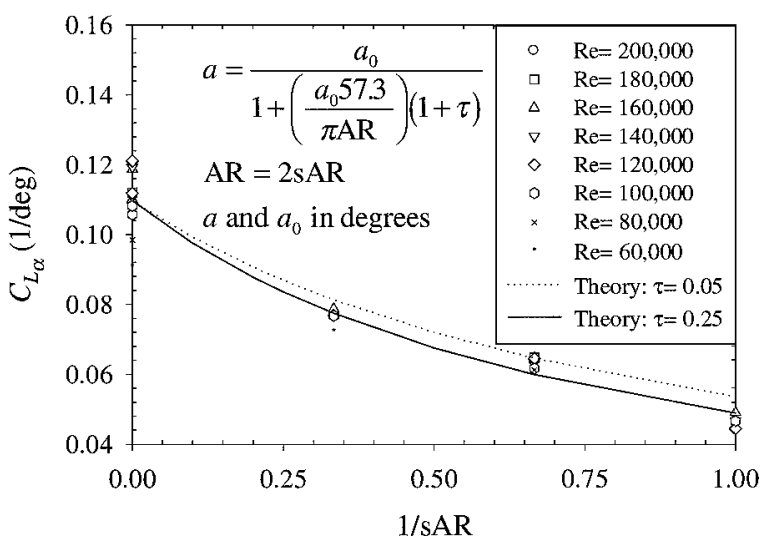

Fig. 9 Lift-curve slope for cambered-plate models. 


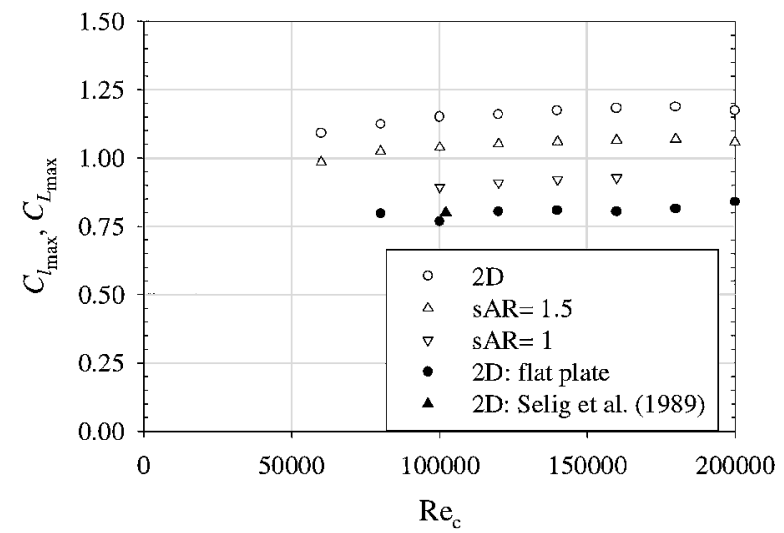

Fig. 10 Maximum lift coefficient as a function of $R e_{c}$ for cambered wings.

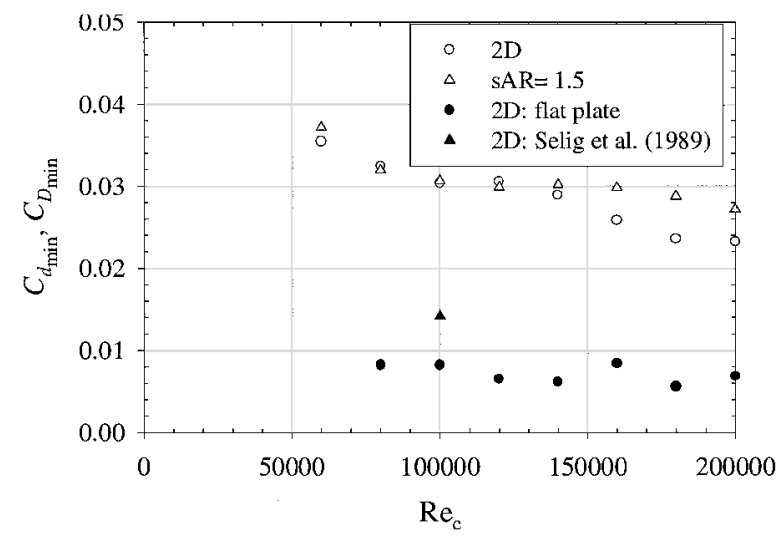

Fig. 11 Minimum drag coefficient as a function of $R e_{c}$ for cambered wings.

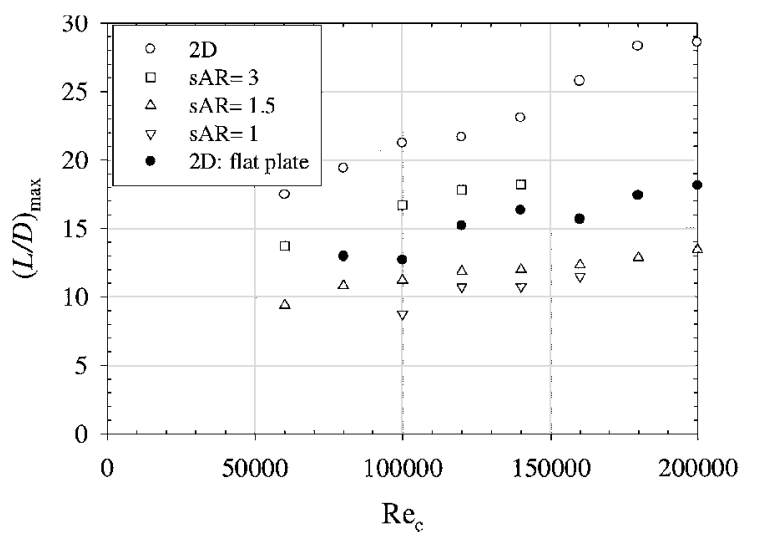

Fig. 12 Maximum $L / D$ ratio as a function of $R e_{c}$ for cambered wings.

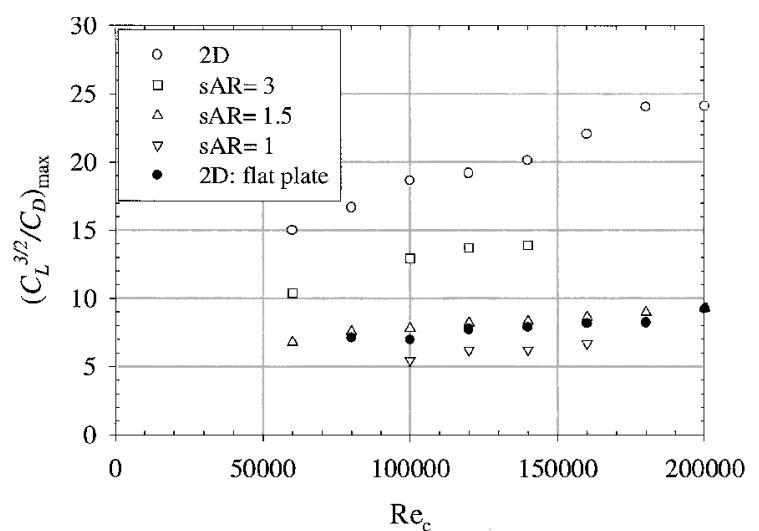

Fig. 13 Maximum $C_{L}^{3 \triangleleft 2} / C_{D}$ ratio as a function of $R e_{c}$ for cambered wings. plates and cambered plates can be compared. Because the cambered wings appear more suitable in the design of MAVs, performance data for only the cambered plates are discussed in more detail. As was just mentioned, Figs. 10-13 show the behavior of $C_{L_{\max }}, C_{D_{\min }}$, $(L / D)_{\max }$, and $\left(C_{L}^{3 / 2} / C_{D}\right)_{\max }$ as a function of Reynolds number for cambered plates of different aspect ratios. The maximum $L / D$ ratio is related to the maximum range for a propeller-driven airplane, whereas the maximum $C_{L}^{3 / 2} / C_{D}$ is related to best endurance(longest flying time possible). In general, when investigatorstry to determine how $C_{L_{\max }}$ and $C_{D_{\min }}$ vary with Reynolds numbers, they determine $C_{L_{\max }}$ and $C_{D_{\min }}$ from $C_{L}$ vs $\alpha$ and $C_{D}$ vs $\alpha$ curves at different Reynolds numbers. In this investigation, the experimental data for $C_{L}$ and $C_{D}$ were fitted with a 10 th-order polynomial, and $C_{L_{\max }}$ and $C_{D_{\min }}$ were obtained from the fitted data. The fitted data were also used to compute $L / D\left(=C_{L} / C_{D}\right)$ and $C_{L}^{3 / 2} / C_{D}$. Fitting the data helped to eliminate large variations resulting from scatter in $C_{D}$ at approximately $\alpha=0 \mathrm{deg}$. As expected, $C_{L_{\max }}$ increased with Reynolds number and aspect ratio in the range of Reynolds numbers tested. The same expected behavior was obtained for $(L / D)_{\max }$ and $\left(C_{L}^{3 / 2} / C_{D}\right)_{\max }$. On the other hand, $C_{D_{\min }}$ showed an increase with decreasing Reynolds number, as was also expected. The maximum $L / D$ generally occurred at $\alpha=3-4 \mathrm{deg}$, whereas $\left(C_{L}^{3 / 2} / C_{D}\right)_{\max }$ occurred at $\alpha=4-5 \mathrm{deg}$, which is a few degrees smaller than what is usually obtained with thicker airfoils/wings. It is important to note that the presence of the end plates usually leads to a larger $C_{D_{\min }}$. For an $18 \%$ thick airfoil (NACA $66_{3}-018$ ), Mueller and Jansen $^{10}$ showed that the interaction between the end plates and the model resulted in a $20 \%$ increase in $C_{d_{\min }}$ at Reynolds numbers between $6 \times 10^{4}$ and $2 \times 10^{5}$. This issue will have to be investigated further to see how the end plates affect $C_{D}$ and hence $L / D$ and $C_{L}^{3 / 2} / C_{D}$ for the thin-wing/airfoil models used in this study.

It was observed in this investigationthat it was sometimes difficult to measure drag accurately because of the difficulty involved in measuring the very small drag forces. A slight offset in one $C_{D}$ vs $\alpha$ curve can lead to jagged $C_{D_{\min }}$ vs $R e_{c}$ curves. Other techniques are being explored to obtain $C_{D_{\min }}$ vs $R e_{c}$. For one of these techniques, the angle of attack is fixed to the angle yielding the lowest $C_{D}$ in a $C_{D}$ vs $\alpha$ curve, and measurements are taken for a series of increasing and decreasing Reynolds numbers without stopping the tunnel. When data are taken for increasing and decreasing Reynolds numbers, the presence of hysteresis can be detected. As mentioned earlier, there was no static hysteresis in the data taken in this investigation.

\section{Effect of Trailing-Edge Geometry}

Four models were tested in the wind tunnel at several chord Reynolds numbers to see if the trailing-edgegeometry had any influence on the aerodynamic characteristics of thin flat plates and cambered plates at low chord Reynolds numbers. The first two models had a tapered trailing edge, and the other two models had an elliptical trailing edge. As mentioned earlier, tests indicated that the shape of the leading-edge geometry (elliptical or sharp) did not appear to influence the results. Results were obtained for infinite models (two-dimensional case) and models with a semispan aspect ratio of $\mathrm{sAR}=1.5$. For the two-dimensional flat-plate case, no significant difference was observed in $C_{L}$ and $C_{D}$ as a function of trailingedge geometry, as shown in Figs. $14 \mathrm{a}$ and $14 \mathrm{~b}$ for $R e_{c}=8 \times 10^{4}$. A difference was, however, observed in the moment coefficient $C_{m / 4}$. For a sharp trailing edge, $C_{m_{\alpha}}$ often appeared to be positive at approximately $\alpha=0 \mathrm{deg}$, even with the uncertainty considered (error bars in $C_{m / 4}$ are approximately the size of the symbols). With the elliptical trailing edge, the two-dimensional cases at $\operatorname{Re}_{c}=8 \times 10^{4}$ showed a stable negative value of $C_{m_{\alpha}}$, as shown in Fig. 14c. For a semispan aspect ratio of $1.5, C_{m_{\alpha}}$ was basically zero at $\alpha=0 \mathrm{deg}$.

With the cambered plates, there was basically no difference between a sharp trailing edge and an elliptical trailing edge at $R e_{c}=$ $8 \times 10^{4}$, as shown in Fig. 15. Results with the cambered plates agree with those of Laitone, ${ }^{11,12}$ who showed that at low Reynolds numbers, a sharp trailing edge is not as critical as for larger Reynolds numbers. 


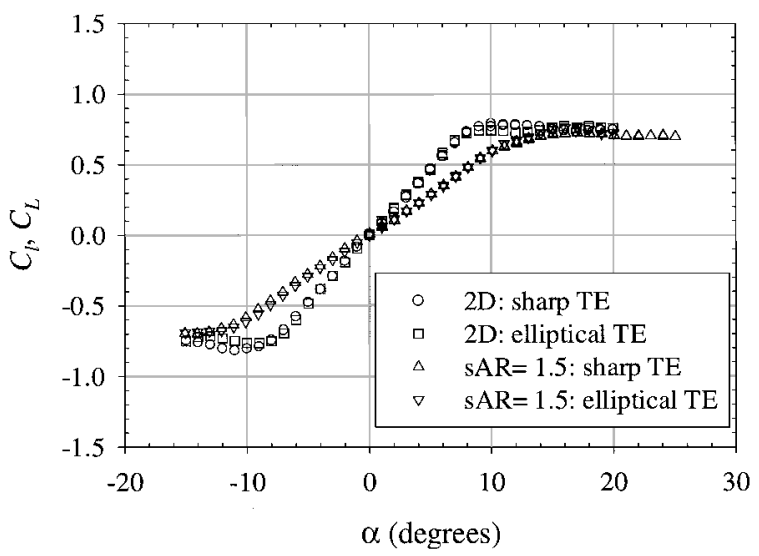

a) Lift coefficient

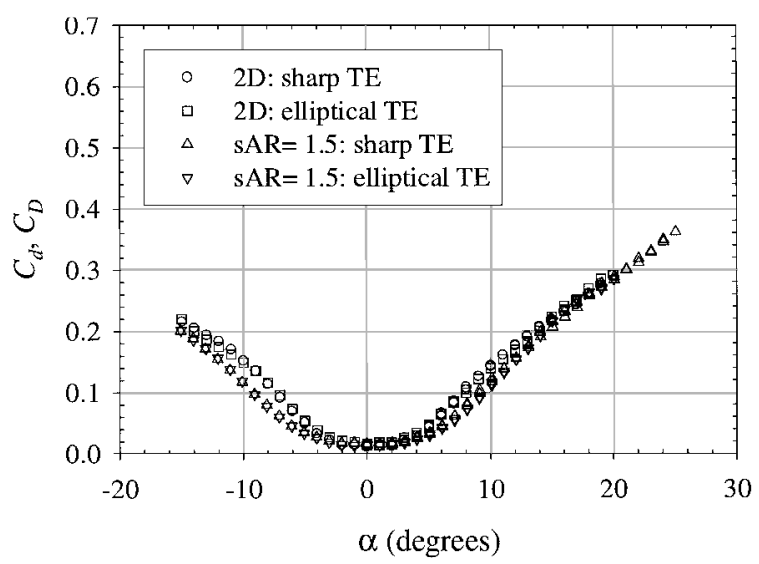

b) Drag coefficient

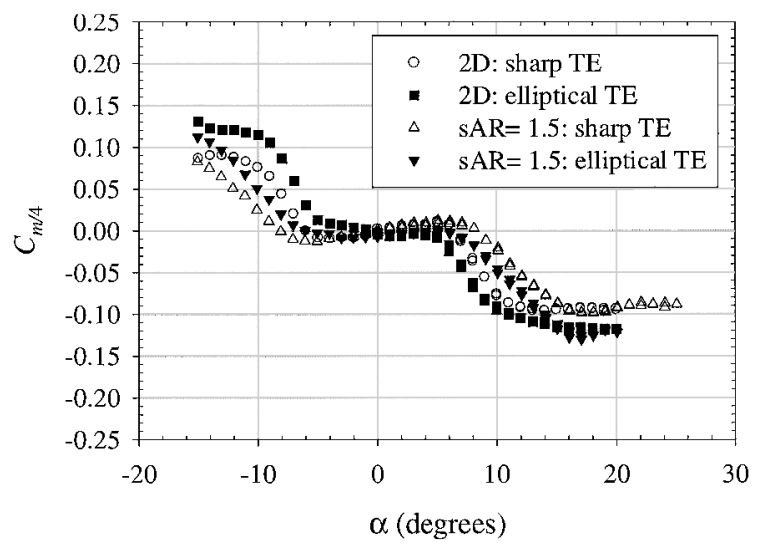

c) Pitching-moment coefficient

Fig. 14 Trailing-edge geometry effect at $R e_{c}=8 \times 10^{4}$ on flat plates.

\section{Effect of Turbulence}

Mueller et al. ${ }^{13}$ showed that an increase in freestream turbulence intensity reduced the minimum drag acting on an $11 \%$ thick Lissaman 7769 airfoil at $R e_{c}=1.5 \times 10^{5}$ and slightly increased $C_{l_{\max }}$. This was caused by an earlier laminar shear layer transition, hence earlier flow reattachment (i.e., a shorter separation bubble) with a larger turbulence intensity. At large angles of attack at which the flow is mostly separated, they observed an increase in drag coefficient with an increase in turbulence intensity. Increasing the turbulence intensity also helped to eliminate some of the hysteresis encountered in $C_{l}$ and $C_{d}$ with their Lissaman airfoil.

Pohle ${ }^{14}$ also looked at the influence of turbulence intensity on a 13\% thick Miley airfoil (M06-13-128) (Ref. 15). He found that increasing the turbulence intensity helped to reduce the hysteresis in $C_{l}$ and $C_{d}$ and slightly improved airfoil performance.

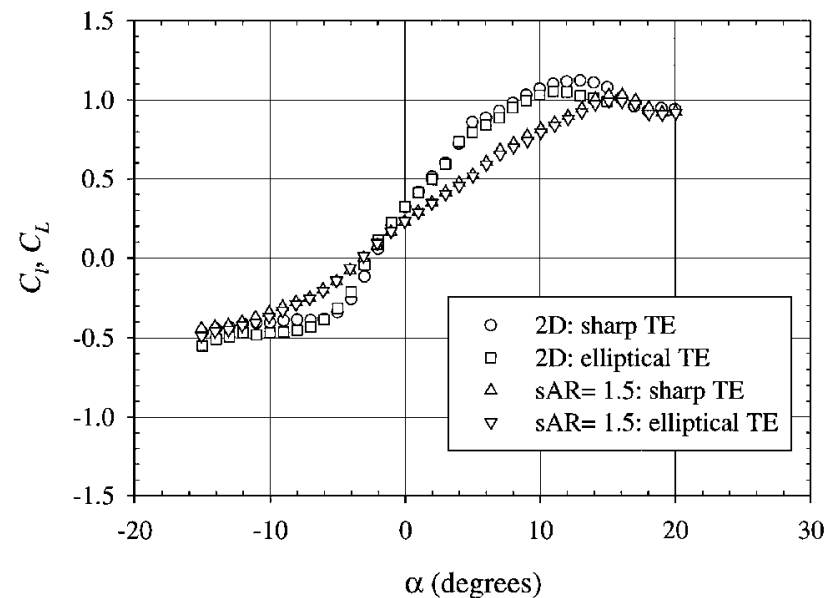

a) Lift coefficient

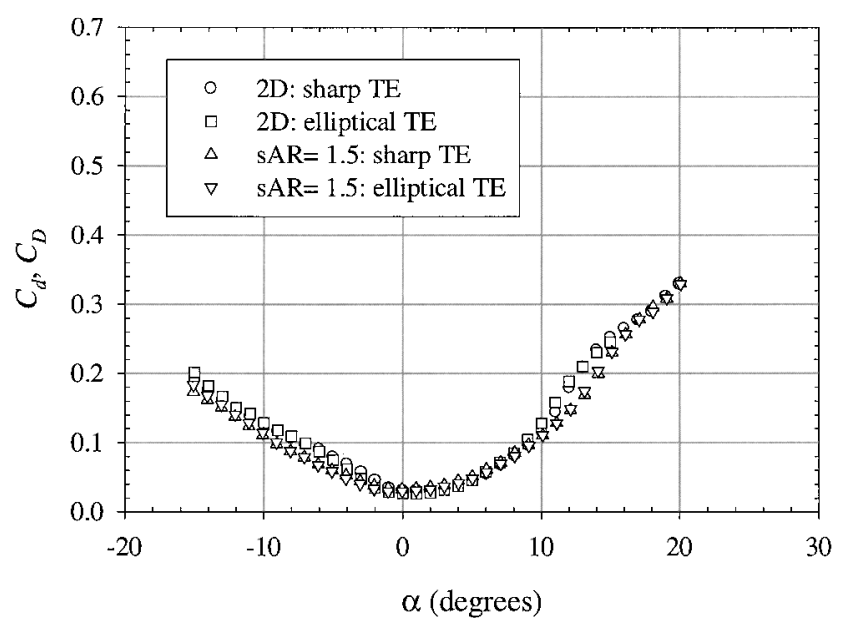

b) Drag coefficient

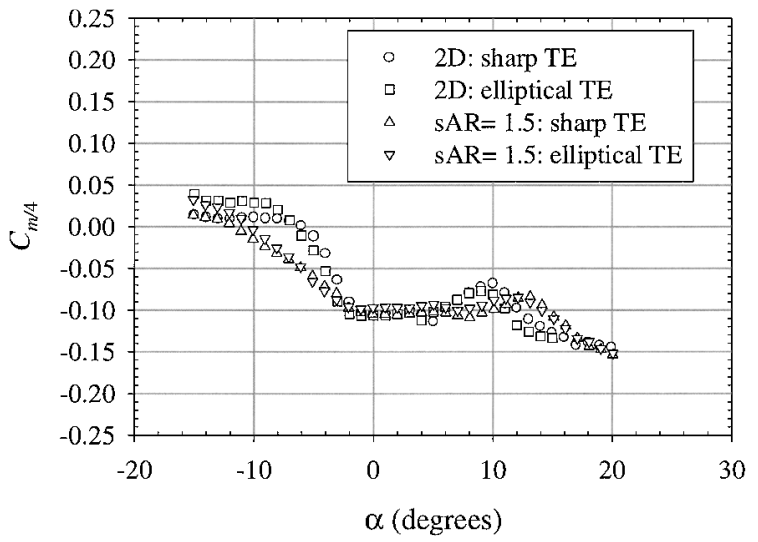

c) Pitching-moment coefficient

Fig. 15 Trailing-edge geometry effect at $R e_{c}=8 \times 10^{4}$ on cambered plates.

Tests were then conducted in the Notre Dame wind tunnel with different turbulence-generatingscreens upstream of the models and a flow restrictor downstream of the model to see if a difference in the turbulence intensity could result in different aerodynamic properties for the models used in this investigation. The flow restrictor, or strawbox, was made of drinking straws packed in a wooden frame and placed between the test section and the diffuser. The flow restrictor was used in only this phase of the investigation. The additional turbulence intensity generated by the strawbox was determined to be approximately $0.05 \%$ (Ref. 16). Table 2 indicates the mesh size and the nominal freestream turbulence intensity in the test section with only a screen present (no flow restrictor). 
Table 2 Turbulence screen data ${ }^{14,16}$

\begin{tabular}{lccc}
\hline \hline Screen & $\begin{array}{c}\text { Mesh size, } \\
\text { meshes/cm }\end{array}$ & $\begin{array}{c}\text { Wire diameter, } \\
\mathrm{mm}\end{array}$ & $\begin{array}{c}\text { Turbulence, } \\
\%\end{array}$ \\
\hline Fine & 7.09 & 0.245 & 0.25 \\
Medium & 3.15 & 0.508 & 0.45 \\
Coarse & 0.64 & 1.397 & 1.3 \\
\hline \hline
\end{tabular}

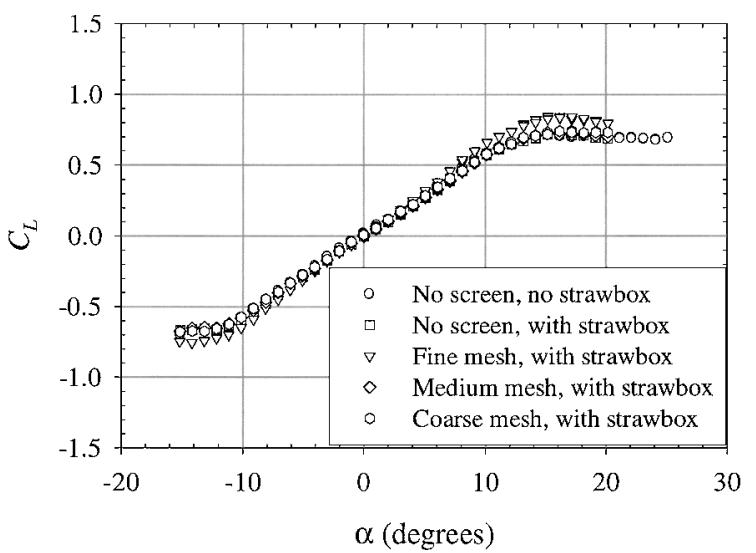

a) Lift coefficient

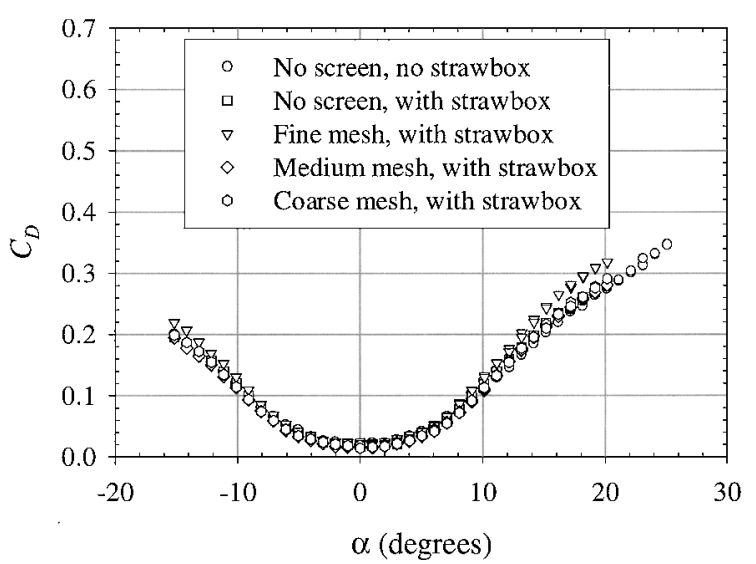

b) Drag coefficient

Fig. 16 Freestream turbulence effect at $R e_{c}=6 \times 10^{4}$ for the $S A R=$ 1.5 flat-plate model.

No measurable differences were observed in the results for different turbulence intensities at $R e_{c}=6 \times 10^{4}$ on the sAR $=1.5$ flatplate model, as shown in Fig. 16. Only a slight increase in $C_{L_{\max }}$ and an increase in $C_{D}$ for large angles of attack is noted for the case with the fine mesh and with the strawbox. All other cases gave the same results. Therefore the effect of turbulenceintensity appeared to be minimal in the wind tunnel for the models tested. Similar results showing a negligible effect of the turbulenceintensity were obtained at $R e_{c}=1.2 \times 10^{5}$ in the wind tunnel and at $R e_{c}=3.9 \times 10^{4}$ and $6 \times 10^{4}$ in a water tunnel. As was mentioned before, flow visualization in the water tunnel did not indicate the presence of flow reattachment after separation. Because there was no flow reattachment after separation for the thin plates tested and because turbulence intensity usually affects the location of this reattachment process and the location of separation, the results were not expected to be strongly affected by varying turbulence intensity. This is exactly the behavior that was observed.

\section{Conclusions}

It has been shown that cambered-plate wings with $4 \%$ camber offer better aerodynamic characteristics than flat-plate wings for a given Reynolds number and aspectratio. No hysteresis was observed in any of the results. Furthermore, reducing the Reynolds number decreases the wing performance, as indicated by large reductions in $(L / D)_{\max }$ and $\left(C_{L}^{3 / 2} / C_{D}\right)_{\max }$. Finally, an increase in turbulence intensity in the wind tunnel and the trailing-edge geometry has been shown to have a very small effect on the measurements of $C_{L}, C_{D}$, and $C_{m / 4}$ on the thin models tested in this investigation.

\section{Acknowledgments}

This research was sponsored by the U.S. Navy, Naval Research Laboratory, Washington, D.C., under Contract 0173-98-C-2025and the Roth-Gibson Endowment at the University of Notre Dame. The authors also acknowledge the assistance of Anthony Fernando, Britt Holewinski, Nicolas Fehring, and Kyle Shaw in obtaining these data.

\section{References}

${ }^{1}$ Wilson, J. R., "Mini Technology for Major Impact," Aerospace America, Vol. 36, No. 5, 1998, pp. 36-42.

${ }^{2}$ Selig, M. S., Donovan, J. F., and Fraser, D. B., Airfoils at Low Speeds, Stokely, Virginia Beach, VA, 1989.

${ }^{3}$ Pankhurst, R. C., and Holder, D. W., Wind-Tunnel Technique, Pitman, London, 1952, pp. 327-427.

${ }^{4}$ Rae, W. H., Jr., and Pope, A., Low-Speed Wind Tunnel Testing, 2nd ed., Wiley, New York, 1984, pp. 176, 344-444.

${ }^{5}$ Mueller, T. J., and Burns, T. F., "Experimental Studies of the Eppler 61 Airfoil at Low Reynolds Numbers," AIAA Paper 82-0345, Jan. 1982.

${ }^{6}$ Huber, A. F., II, "The Effects of Roughness on an Airfoil at Low Reynolds Numbers," M.S. Thesis, Dept. of Aerospace and Mechanical Engineering, Univ. of Notre Dame, IN, May 1985.

${ }^{7}$ Mueller, T. J., Batill, S. M., Brendel, M., Perry, M. L., Bloch, D. R., Huber, A. F., II, Bastedo, W. G., Jr., O'meara, M. M., and Schmidt, G. S., "Low Reynolds Number Wind Tunnel Measurements: The Importance of Being Earnest," Aerodynamics at Low Reynolds Numbers $10^{4}<R e<10^{6}$ International Conference, Royal Aeronautical Society, London, 1986, pp. $14.1-14.18$

${ }^{8}$ Kline, S. J., and McClintock, F. A., "Describing Uncertainties in SingleSample Experiments," Mechanical Engineering, Vol. 75, No. 1, 1953, pp. 3-8.

${ }^{9}$ Anderson, J. D., Jr., Fundamentals of Aerodynamics, 2nd ed., McGrawHill, New York, 1991, p. 343.

${ }^{10}$ Mueller, T. J., and Jansen, B. J., Jr., "Aerodynamic Measurements at Low Reynolds Numbers," AIAA Paper 82-0598, March 1982.

${ }^{11}$ Laitone, E. V., "Aerodynamic Lift at Reynolds Numbers Below $7 \times 10^{4}, "$ AIAA Journal, Vol. 34, No. 9, 1996, pp. 1941, 1942 .

${ }^{12}$ Laitone, E. V., "Wind Tunnel Tests of Wings at Reynolds Numbers Below 70,000," Experiments in Fluids, Vol. 23, No. 5, 1997, pp. 405-409.

${ }^{13}$ Mueller, T. J., Pohlen, L. J., Conigliaro, P. E., and Hansen, B. J., Jr., "The Influence of Free-Stream Disturbances on Low Reynolds Number Airfoil Experiments," Experiments in Fluids, Vol. 1, No. 1, 1983, pp. 3-14.

${ }^{14}$ Pohlen, L., "Experimental Studies of the Effect of Boundary Layer Transition on the Performance of the Miley (M06-13-128) Airfoil at Low Reynolds Numbers," M.S. Thesis, Univ. of Notre Dame, IN, Jan. 1983.

${ }^{15}$ Miley, S. J., "An Analysis of the Design of Airfoil Sections for Low Reynolds Numbers," Ph.D. Dissertation, Dept. of Aeronautical and Astronautical Engineering, Mississippi State Univ., Starkeville, MS, 1972.

${ }^{16}$ Brendel, M., and Huber, A. F., II, "An Experimental Investigation of Flow Quality in an Indraft Subsonic Wind Tunnel Using a Single Hot Wire Anemometer," Univ. of Notre Dame, IN, Nov. 1984. 